\title{
Custos do Programa de Gerenciamento de Doentes Crônicos de uma operadora de saúde
}

\author{
Costs of Chronic Patient Management Program in a healthcare provider organization \\ Los costos del Programa de Gestión de Pacientes Crónicos en una opertadora de salud
}

\author{
Sarah Lopes Silva'; Ana Clara Tolentino"'; Luis Carlos Santiago "II; Cristiano Bertolossi Martalv; \\ Antônio Augusto de Freitas Peregrinovi ${ }^{v}$ Vivian Schutz ${ }^{\text {VI }}$
}

\begin{abstract}
RESUMO
Objetivo: analisar a estratégia de contenção de custos por múltiplas internações evitáveis de um Programa de Gerenciamento de Doentes Crônicos (GDC), desde sua implantação, em uma operadora de saúde. Método: estudo retrospectivo, abordagem quantitativa em que foi realizado um levantamento dos dados obtidos através do acompanhamento dos 243 pacientes assistidos pelo programa de 2009 a 2012. Aprovado pelo CEP sob o CAAE: 34525614.8 .0000 .5285 . Resultados: com relação às patologias de base, a maioria dos pacientes possuiu mais de uma doença instalada. Com relação aos custos decorrentes de internação de emergência no ano anterior ao GDC foi de $\mathrm{R} \$ 1.323 .295,43$ e no ano posterior $\mathrm{R} \$ 629.565,89$. Foi possível observar o quão custosos foram os pacientes que apresentaram as patologias possíveis de serem evitadas. Conclusão: percebe-se que uma gestão eficiente em saúde possibilita oferecer serviços para um número maior de usuários, sem prejudicar as operadoras, em especial quando alocados em ações envolvendo a prevenção. Palavras-chave: Custo e análise de custos; saúde suplementar; doença crônica; envelhecimento.
\end{abstract}

\section{ABSTRACT}

Objective: to analyze the cost-containment strategy for multiple avoidable hospitalizations in a program of Chronic Patient Management (GDC), since its implementation in a health care provider organization. Method: this is a retrospective study with quantitative approach in which a survey was conducted with data obtained through monitoring of 243 patients assisted by the program from 2009 to 2012. Approved by Research Ethics Comitee (CAAE: 34525614.8.0000.5285). Results: in the case of basic conditions, most patients possessed more than one installed disease. Regarding the costs of emergency hospitalization in the year before GDC was BRL $1,323,295.43$ and the subsequent year BRL 629,565.89. It was possible to observe how expensive were the patients who presented pathologies that could be avoided. Conclusion: it was concluded that an efficient management in health allows profesionals to offer services in order to a greater number of users with no prejudice to the operators, especially when allocated to preventive actions. Keywords: Cost and cost analysis; supplemental health; chronic disease; aging.

\section{RESUMEN}

Objetivo: analizar la estrategia de contención de costos de múltiples hospitalizaciones evitables en un programa de Manejo de Pacientes Crónicos (GDC), desde su implementación en una organización de proveedores de atención médica. Método: se trata de un estudio retrospectivo con enfoque cuantitativo en el que se realizó una encuesta con datos obtenidos a través del seguimiento de 243 pacientes atendidos por el programa de 2009 a 2012. Aprobado por el Comité de Ética en Investigación (CAAE: 34525614.8.0000.5285). Resultados: en el caso de condiciones básicas, la mayoría de los pacientes poseían más de una enfermedad instalada. En cuanto a los costos de hospitalización de emergencia en el año anterior a la GDC fue de R \$1.323.295,43 y el año siguiente BRL 629,565.89. Se pudo observar cuán costosos eran los pacientes que presentaban patologías que podían evitarse. Conclusión: se concluyó que una gestión eficiente en salud permite a los profesionales ofrecer servicios a un mayor número de usuarios sin perjuicio de los operadores, especialmente cuando se les asignan acciones preventivas.

Palabras clave: Costos y análisis de costos; seguro de salud; enfermedad crónica; envejecimiento.

\section{INTRODUÇÃO}

O Brasil vem sofrendo uma transição epidemiológica nas últimas décadas, caracterizada pelo aumento de doenças crônico-degenerativas e declínio das doenças infecto-parasitárias, ocasionadas pelos avanços em tecnologias e ciências da saúde. Essa mudança no perfil de saúde populacional contribui significativamente para a redução da mortalidade, o que leva ao aumento da expectativa de vida da população brasileira ${ }^{1-3}$.

Estimativas apontam que em 2050, a população mundial com idade superior a 60 anos seja de cerca de

'Mestranda em Enfermagem, Universidade Federal do Estado do Rio de Janeiro. Brasil. E-mail: enfasarah@gmail.com

"Doutoranda em enfermagem, Universidade Federal do Estado do Rio de Janeiro. Brasil. Email: anaclaratolentino@gmail.com

II'Pós Doutor. Professor Adjunto do departamento de enfermagem fundamental da Universidade Federal do Estado do Rio de Janeiro. Brasil. E-mail: luisolitrio@yahoo.com.br IV Pós Doutor em enfermagem. Professor Adjunto do departamento de fundamentos de enfermagem da Universidade do Estado do Rio de Janeiro. Brasil. E-mail: cristianobertol@gmail.com

v Doutor em saúde coletiva. Professor Adjunto do Laboratório de Ciências Radiológicas da Universidade do Estado do Rio de Janeiro.Brasil. E-mail: antoniop@uerj.br v' Doutora em enfermagem. Professora adjunta do departamento de enfermagem fundamental da Universidade Federal do Estado do Rio de Janeiro. Brasil. E-mail: vshutz@gmail.com 
dois bilhões de pessoas. Isso ocorrerá como resultado à mudança nos indicadores de saúde relacionados à queda na taxa de fecundidade e mortalidade em oposição ao aumento da expectativa de vida ${ }^{4}$.

Com o envelhecimento populacional ocorre, consequentemente, o aumento das doenças crônicas, que possuem desenvolvimento lento e longa duração, e tem sido motivo da preocupação de muitos gestores de saúde, pois ocasionam aumentos significativos nos custos em saúde em todo o mundo por demandarem tratamentos complexos e de longa duração, interferindo nos orçamentos públicos e privados. De acordo com o World Economic Forum, entre 2011 e 2030 as perdas econômicas geradas pelas doenças crônicas não transmissíveis serão de até 47 trilhões de dólares, montante que representa $5 \%$ do produto global bruto desse período. No Brasil não será diferente e até o ano de 2015 espera-se um custo de US\$4,18 bilhões gastos com diabetes, doença cardiovascular e acidente vascular encefálico ${ }^{5,6}$.

Entre as doenças crônicas que mais acometem a população idosa, estão as patologias cardiovasculares, a doença pulmonar obstrutiva crônica (DPOC) e o Diabetes Mellitus (DM). Estas podem estar relacionadas não somente ao processo do envelhecimento, mas também aos hábitos de vida como consumo de tabaco e álcool, alimentação, sedentarismo e predisposição genética. Entretanto, essas doenças atualmente não acometem somente a população idosa e os países ricos, mas também os jovens e pessoas de meia-idade vivendo em países em desenvolvimento ${ }^{6,7}$.

Atualmente, esforços sugerem a adoção de programas de promoção e envelhecimento saudável. O Ministério da Saúde incluiu como item prioritário no sistema de saúde do país, a saúde do idoso. A própria Agência Nacional de Saúde Suplementar (ANS), através da Resolução Normativa ${ }^{\circ} 265$, incentiva a participação de usuários de planos de saúde em programas de envelhecimento ativo. Entretanto, o foco assistencial ainda é preponderante e um desafio a ser superado constantemente ${ }^{2,8}$.

Por outro lado, existem os pacientes com patologias já instaladas. Doenças crônicas não regridem, portanto deve-se desacelerar sua progressão, prolongando a expectativa de vida ativa. Nesse contexto faz-se necessário um programa para cuidar dos idosos e suas múltiplas patologias associadas, através de grupos de gerenciamento de pacientes portadores de doenças crônicas ${ }^{8}$.

Este estudo objetivou analisar a estratégia de contenção de custos por múltiplas internações evitáveis. Essa estratégia foi adotada por uma operadora de saúde de autogestão, após a implantação do programa de gerenciamento de doentes crônicos (GDC). Este programa foi implantado em 2009, e vem sofrendo alterações constantes. Esse objetivo vem responder ao seguinte questionamento: Houve redução nos custos gerados pelos pacientes acompanhados por esse programa no ano posterior à sua implantação?
O público alvo desse programa são portadores de doenças crônico-degenerativas como DPOC, DM, hipertensão arterial sistêmica (HAS), doença coronariana (DC), dislipidemia (DLP) e insuficiência cardíaca congestiva (ICC), selecionados por critérios pré-estabelecidos: presença de no mínimo uma das patologias de base supracitadas e internações decorrentes desses agravos.

Estes pacientes são acompanhados por uma equipe multidisciplinar, através de ligações telefônicas programadas, de acordo com o grau de complexidade em que se enquadram, podendo ser: leve, moderada ou grave. Essas ligações podem ser trimestrais, mensais ou quinzenais, respectivamente. Esse acompanhamento é feito por uma empresa terceirizada que repassa os relatórios conforme a periodicidade acordada. A operadora por sua vez, paga um valor per capita, por associado, de acordo com o grau de complexidade acima descrito.

\section{REVISÃO DE LITERATURA}

O mercado de saúde suplementar no Brasil é subdividido em seguradoras, cooperativas, medicinas de grupo e operadoras de autogestão. Estas correspondem a um segmento não comercial e, portanto, sem fins lucrativos, da atenção à saúde e que em alguns casos contam com o patrocínio das empresas empregadoras. A lógica de mercado não se emprega a essas operadoras por não visarem lucros, sendo as receitas revertidas no custeio da própria atividade ${ }^{9}$.

Os custos em saúde vêm aumentando exponencialmente, ocasionando a escassez dos recursos disponíveis, o que coloca as fontes pagadoras, públicas ou privadas, em situação delicada. Torna-se necessária, portanto, a aquisição de conhecimentos gerenciais que contribuam para a restrição de custos e alocação eficaz de recursos a fim de manter o equilíbrio financeiro das operadoras de saúde frente a esse panorama ${ }^{10,11}$.

Diante deste cenário, é fundamental que os gestores do setor saúde mantenham foco na criação de valores e não apenas no corte de custos. Portanto, faz-se necessária a adoção de medidas preventivas e de um programa de gerenciamento abrangente a fim de reduzir o risco e agravo das enfermidades. Estudos apontam que é possível obter o retorno de US\$2,9 para cada dólar investido em gerenciamento de doenças crônicas e prevenção ${ }^{1}$.

Algumas operadoras, atualmente, na tentativa de controle de custos, iniciaram programas de atenção a pacientes crônicos com o intuito de identificá-los e acompanhá-los regularmente. Entretanto, poucas são as publicações em que se mostram os benefícios do programa no que se refere a este custo.

Esses programas possuem como finalidade identificar indivíduos portadores de patologias crônicas de alto risco assistencial, atuando na prevenção de seus agravos através do envolvimento do mesmo e seus fa- 
miliares no cuidado, além de gerar uma base de dados sobre esses doentes. A definição adequada da forma de atuação e a capacidade da equipe multidisciplinar responsável por esse programa é de extrema importância para seu sucesso ${ }^{1}$.

\section{Metodologia}

Trata-se de um estudo retrospectivo, de abordagem quantitativa. Para a resolução da questão de estudo foi realizado um levantamento dos dados obtidos através do acompanhamento dos pacientes assistidos pelo programa GDC desde 2009, através da análise do banco de dados de uma operadora privada de autogestão, localizada no estado do Rio de janeiro. Esse programa sofreu mudanças em sua gestão no ano de 2013. A coleta de dados ocorreu entre os meses de novembro e dezembro de 2014.

O programa teve inicio em 2009 contando com a participação de 78 associados, selecionados a partir dos históricos de internações e patologias de base. Novos associados foram incluídos progressivamente, a partir das pesquisas de atendimentos e internações de pacientes portadores das patologias de base anteriormente citadas. No primeiro semestre de 2010 foram incluídos 10 novos associados, no segundo semestre de 2011, 30 novos pacientes foram integrados e em 2012, 71 no primeiro semestre e 61 no segundo, totalizando 250 integrantes.

Uma nova busca por associados vem ocorrendo atualmente através da identificação de pacientes com múltiplas internações e portadores das patologias de base, entretanto esses novos associados não são elegíveis a esse estudo por não possuírem um ano no programa.

Os critérios de inclusão no estudo foram: pacientes que estavam em acompanhamento pelo programa no momento da coleta de dados e terem completado 12 meses de adesão ao mesmo.

Durante o período de acompanhamento, sete associados foram desligados do GDC, sendo excluídos do estudo. A população foi composta por 243 participantes, o que representa todos os associados que compõe o programa, vale ressaltar que esse programa é de abrangência nacional, portanto conta com associados de todas as regiões.

Para atender aos objetivos deste estudo, foram percorridas as seguintes etapas:

- Foram selecionados os pacientes que se enquadravam nos critérios de inclusão, através da análise do banco de dados adotado pela operadora. Os pacientes selecionados foram divididos de acordo com o ano de adesão ao programa, de 2009 a 2012;

- Foi realizado um levantamento do valor pago em reais à empresa prestadora de serviço por cada associado de acordo com o nível em que se enquadra e o valor total;
- Foram identificadas doenças de bases de cada associado, faixa etária e sexo. Esses dados foram obtidos através do software Benner Solution ${ }^{\circledR} 2006$, da empresa responsável pelo desenvolvimento e manutenção desse programa;

- Cada histórico foi analisado no período de 12 meses antes da adesão e 12 meses após no que diz respeito à presença de internações de emergência, valor total das internações por ano e presença de atendimentos de urgência. Para tal, foi realizada uma consulta no banco de dados da empresa, que conta com um sistema open base versão 01/2006, e através do número de matrícula do associado foi possível obter todas as senhas geradas para internações, assim como o valor pago ao prestador executante de tal internação e também o número de atendimentos de urgência/emergência.

Os dados foram organizados extraídos para o Microsoft Access $2007{ }^{\circledR}$ e organizados através de tabelas.

Vale ressaltar que o acesso ao banco de dados foi autorizado pelo diretor de previdência e assistência através da assinatura de um termo de anuência. Não houve encaminhamento ao Comitê de Ética por não apresentar risco aos sujeitos envolvidos nem exposição das identidades.

\section{RESULTADOS E DISCUSSÃo}

Os associados iniciaram o acompanhamento no momento de inclusão no grupo de doentes crônicos e a partir dessa etapa cada paciente recebeu uma classificação relacionada à doença de base de maior gravidade. A partir dessa avaliação foi definido a alocação em cada grupo.

Com relação à classificação por grupos, 178 $(73,2 \%)$ pertenciam do grupo $1,57(23,4 \%)$ do grupo 2 e $8(3,2 \%)$ do grupo 3, totalizando 243 associados. Durante o ano de acompanhamento, 4 (1,6\%) evoluíram para óbito e $3(1,2 \% \%)$ obtiveram alta melhorada, sendo excluídos do estudo sete pacientes.

Dados na literatura apontam para modelos teóricos aplicados aos idosos de acordo com seu grau de dependência e complexidade, para a estratificação em quatro níveis a fim de nortear as ações a serem empregadas em cada grupo em programas de promoção de saúde e controle de doenças. Esses modelos partem da lógica de que esses indivíduos devem ser acompanhados permanentemente de acordo com a periodicidade respectiva do grupo ao qual pertencem².

Quanto ao sexo, não existiu grande diferença entre a prevalência de homens e mulheres, sendo $53 \%$ mulheres e $47 \%$ homens. Com relação à faixa etária, a maioria dos associados possuia idade entre 61 e 80 anos (67\%) seguida pelos associados com idade acima de 80 anos (20\%), fato esperado por tratar-se de portadores de doenças crônicas com maior prevalência em idosos. 
No que diz respeito às patologias de base, a maioria dos pacientes possuía mais de uma doença instalada, sendo grande parte hipertensos (93\%), seguidos de dislipidêmicos (51\%), DM (47\%), portadores de DPOC (42\%), DAC (5\%) e ICC (4\%).

A hipertensão arterial sistêmica representa uma das maiores causas de redução na expectativa e qualidade de vida dos indivíduos e está diretamente relacionada ao desenvolvimento de agravos cardiovasculares, sendo responsável por $25 \%$ da etiologia multifatorial da cardiopatia isquêmica e $40 \%$ dos acidentes vasculares cerebrais. No Brasil, as doenças cardiovasculares são as maiores causas de internações no setor público e responsáveis por $33 \%$ dos óbitos por causas conhecidas. A maioria dessas doenças ocorre como desfecho de portadores de fatores de risco de grau leve, sem o devido tratamento, como por exemplo, os hipertensos ${ }^{12-14}$.

O DM, no ano de 2010 , atingiu $6,4 \%$ de adultos entre 20 e 79 anos e em 2008 foi responsável por 1,3 milhões de mortes no mundo. No Brasil esse cenário não é diferente, ocorrendo 54.800 óbitos em um contingente de 6 milhões de portadores. Aliado à alta prevalência de portadores de DM, tem sido observado um crescente aumento nas hospitalizações por essa doença em detrimento de outras ${ }^{15,16}$.

A DPOC caracteriza-se por uma obstrução crônica e progressiva do fluxo expiratório e está associada à exposição ao tabaco e poluentes ambientais além de variações climáticas. No Brasil, esta doença representa a terceira causa de morte por doenças crônicas não transmissíveis estimando-se uma mortalidade anual de 40.000 habitantes. Em 2011, o Sistema Único de Saúde registrou um custo de 103 milhões de reais referente a 142.635 internações por DPOC ${ }^{17}$.

A empresa prestadora de serviços possui um custo de acompanhamento mensal, por associado, de acordo com o grupo ao qual pertence. Conforme o perfil dos associados apresentados, a maioria se enquadrou no grupo 1.Vale ressaltar que apesar do valor por associado ser menor no grupo 1, o custo final com esse grupo é maior em relação aos outros, conforme mostra a Tabela 1.

TABELA 1: Custo (em reais) do programa para a empresa por grupo de associados. Rio de Janeiro, 2014.

\begin{tabular}{cccc}
\hline Grupo & Unitário & $\begin{array}{c}\text { Valor total } \\
\text { mês }\end{array}$ & $\begin{array}{c}\text { Valor total } \\
\text { ano }\end{array}$ \\
\hline 1 & $\mathrm{R} \$ 16,00$ & $\mathrm{R} \$ 2.848,00$ & $\mathrm{R} \$ 34.176,00$ \\
2 & $\mathrm{R} \$ 26,00$ & $\mathrm{R} \$ 1.482,00$ & $\mathrm{R} \$ 17.784,00$ \\
3 & $\mathrm{R} \$ 30,00$ & $\mathrm{R} \$ 240,00$ & $\mathrm{R} \$ 2.880,00$ \\
Total & - & $\mathrm{R} \$ 4.570,00$ & $\mathrm{R} \$ 54.840,00$ \\
\hline
\end{tabular}

A fim de mensurar os custos, os indivíduos foram separados em grupos de acordo com o período em que foram incluídos no programa. Realizou-se um somatório do custo anual dos indivíduos de cada grupo e os dados obtidos foram organizados na Tabela 2

TABELA 2: Custo (em reais) total por internações e número de atendimentos. Rio de Janeiro, 2014.

\begin{tabular}{|c|c|c|c|}
\hline Período de inclusão & Pacientes (f) & $\begin{array}{l}\text { Custo ano } \\
\text { anterior }\end{array}$ & $\begin{array}{l}\text { Custo ano } \\
\text { posterior }\end{array}$ \\
\hline $2^{\circ}$ semestre de 2009 & 78 & $546.698,91$ & 337784,92 \\
\hline $1^{\circ}$ semestre de 2010 & 10 & - & - \\
\hline $2^{\circ}$ semestre de 2011 & 29 & 110840,46 & 30623,08 \\
\hline $1^{\circ}$ semestre de 2012 & 68 & 532983,09 & 66768,23 \\
\hline $2^{\circ}$ semestre de 2012 & 58 & 132772,97 & 194389,66 \\
\hline Total & 243 & $1.323 .295,43$ & 629565,89 \\
\hline Período de inclusão & Pacientes (f) & $\begin{array}{c}\text { Internação ano } \\
\text { anterior }\end{array}$ & $\begin{array}{c}\text { Internação ano } \\
\text { posterior }\end{array}$ \\
\hline $2^{\circ}$ semestre de 2009 & 78 & 47 & 40 \\
\hline $1^{\circ}$ semestre de 2010 & 10 & - & - \\
\hline $2^{\circ}$ semestre de 2011 & 29 & 11 & 5 \\
\hline $1^{\circ}$ semestre de 2012 & 68 & 43 & 22 \\
\hline $2^{\circ}$ semestre de 2012 & 58 & 10 & 12 \\
\hline Total & 243 & 111 & 79 \\
\hline Período de inclusão & Pacientes (f) & $\begin{array}{l}\text { Atendimento } \\
\text { ano anterior }\end{array}$ & $\begin{array}{c}\text { Atendimento ano } \\
\text { posterior }\end{array}$ \\
\hline $2^{\circ}$ semestre de 2009 & 78 & 161 & 103 \\
\hline $1^{\circ}$ semestre de 2010 & 10 & - & - \\
\hline $2^{\circ}$ semestre de 2011 & 29 & 31 & 41 \\
\hline $1^{\circ}$ semestre de 2012 & 68 & 155 & 127 \\
\hline $2^{\circ}$ semestre de 2012 & 58 & 42 & 60 \\
\hline Total & 243 & 389 & 331 \\
\hline
\end{tabular}


Percebe-se, através dos dados, que houve uma redução de aproximadamente $52 \%$ dos custos com internação ano posterior. Se somar ao valor do custo, após a implantação do programa (R\$629.565,89), o custo com a manutenção do mesmo ( $R \$ 54.800,00)$, o valor total alcança $\mathrm{R} \$ 684.365,89$, o que representa o custo com internação acrescido do custo com o programa. Portanto, o percentual total de redução nos custos, a partir desse valor corrigido, foi de aproximadamente $48 \%$, conforme tabela 2 .

Do número total de associados, somente 17 (7\%), obtiveram um custo com internação maior no ano posterior ao tratamento, ou seja, 226 (93\%) pacientes obtiveram redução nos custos.

Com relação ao número de internações, também houve redução no ano posterior ao a GDC. No ano anterior à inclusão, os 243 associados totalizaram 111 internações e no ano posterior esse número foi reduzido para 79, conforme demonstrado na Tabela 2.

Além da obtenção de dados relacionados aos custos com internações e o número das mesmas, foram também identificados os números de atendimentos de urgências e emergência que representam outra fonte de escoamento de recursos para as operadoras em saúde para portadores de doenças crônicas descompensadas, que não fazem o devido acompanhamento como observado na Tabela 2.

No respectivo ano anterior à inclusão dos associados no GDC, o total de atendimentos de urgência e emergência foi de 389 e no ano posterior de 331. Vale ressaltar que em dois grupos houve aumento do número de atendimentos mesmo com o acompanhamento pelo GDC. Ao analisar cada dado separadamente, verificou-se que o aumento foi aleatório e pulverizado nos diversos associados.

\section{CONCLUSÃO}

O gerenciamento de pacientes crônicos representa uma importante ferramenta para uma gestão eficiente dos recursos financeiros em saúde, tão escassos na atualidade. A partir dos dados apresentados, observou-se o quão custosos foram os pacientes que apresentaram as patologias possíveis de serem evitadas e como houve uma redução significativa nos custos com internações para a operadora de saúde, além de redução no número de atendimentos de urgência/emergência e de internações, com a implementação desse programa.

Diante dos fatos mencionados, percebe-se que uma gestão eficiente em saúde possibilita oferecer serviços em saúde para um número maior de usuários, sem prejudicar as operadoras, em especial quando alocados em ações envolvendo a prevenção.

Como limitações deste estudo, pode-se considerar o tempo de acompanhamento dos associados e a mudança na empresa prestadora de serviços durante o período de acompanhamento.
Outros estudos serão necessários para observar a adequação dessa prática em outros tipos de usuários, como por exemplo, o sistema de saúde público.

\section{REFERÊNCIAS}

1. Veras RP. Gerenciamento de doença crônica: equívoco para o grupo etário dos idosos. Rev Saude Publica. 2012; 46(6):929-34. 2. Daros RF, Gomes RS, Silva FH, LOPES TC. A satisfação do beneficiário da saúde suplementar sob a perspectiva da qualidade e integralidade. Physis [online]. 2016; 26(2),525-47.

3. Sousa SPO, Branca SBP. Panorama epidemiológico do processo de envelhecimento no mundo, Brasil e Piauí: evidências na literatura de 1987 a 2009. Enfermagem em Foco. 2011; 2(3):188-90. 4. Ministério da Saúde (Br). Secretaria de Atenção à Saúde. Departamento de Atenção Básica. Envelhecimento e Saúde da Pessoa Idosa. Brasília (DF), 2006. [citado em 05 mar 2016]. Disponível em http://189.28.128.100/dab/docs/publicacoes/cadernos_ab/ abcad19.pdf.

5. Silva LS, Cotta RMM, Rosa COB. Estratégias de promoção da saúde e prevenção primária para enfrentamento de doenças crônicas: revisão sistemática. Rev Panam Salud Publica. 2013; 34(5): 343-50.

6. Veras RP. Estratégias para o enfrentamento das doenças crônicas: um modelo em que todos ganham. Rev Bras Geriatr Gerontol. 2011; 14(4): 779-86.

7. Pimenta MA, Castanheira CHA, Lana FCF, Malta DC. Ações de prevenção de riscos e doenças no setor suplementar. Rev Min Enferm. 2012; 16(4): 564-71.

8. Gerschman S, Ugá MAD, Portela M, Lima SML. O papel necessário da Agência Nacional de Saúde Suplementar na regulação de planos de saúde e prestadores de serviço. Physis Rev de Saúde Coletiva. 2012; 22(2): 463-76.

9. Freitas RM, Cherchiglia ML, Lana FCF, Malta DC, Andrade EIG. Estudo dos modelos assistenciais praticados por operadoras de planos privados de saúde. Physis Revista de Saúde Coletiva. 2011; 21 (4): 1561-77.

10.Lima AFC, Castilho V, Fugulin FMT, Silva B, Ramin NS, Melo TO. Custos das atividades de enfermagem realizadas com maior frequência em pacientes de alta dependência. Rev Latino-Am Enfermagem. 2012 [citado em 05 mar 2016]. 20(5): 880-7. Disponível em: http://www.revistas.usp.br/rlae/article/view/48627/52668. 11. Martins AVF, Peter MGA, Machado MVV, Catrib AMF. Estratégias para a redução de custos em operadoras de plano de saúde. Rev de Informação Contábil. 2013; 7(1): 55-72.

12. Marta CB, Leite JL, Peregrino AAF, Schutz V, Francisco MTR, Magnago C. Custos da adesão ao tratamento da Síndrome da Imunodeficiência Adquirida: em estudo transversal. Rev enferm UERJ. 2014; 22(2):193-9.

13. Silva SSBE, Oliveira SFSB, Pierin AMG. O controle da hipertensão arterial em mulheres e homens: uma análise comparativa. Rev esc enferm USP. 2016; 50(1):50-8.

14. Pinto NBF, Vieira LB, Pereira FMV, Reis AMM, Cassini SHDB. Interações medicamentosas em prescrições de idosos hipertensos: prevalência e significância clínica. Rev enferm UERJ. 2014; 22(6):735-41.

15. Malta DC, Iser BPM, Andrade SSCA, Moura L, Oliveira TP, Bernal RTI. Tendência da prevalência do diabetes melito autorreferido em adultos nas capitais brasileiras, 2006-2012. Rev Epidemiol Serv Saúde. 2014; 23(4): 753-60.

16. Petermann XB, Machado IS, Pimentel BN, Miolo SB, Martins LR, Fedosse E. Epidemiologia e cuidado à Diabetes Mellitus praticado na Atenção Primária à Saúde: uma revisão narrativa. Saúde, Santa Maria. 2015; 41(1): 49-56.

17. Rabahi M. Epidemiologia da DPOC: enfrentando desafios. Rev Pulmão RJ. 2013; 22(2): 4-8. 